
\title{
28 Research Square \\ Evaluating Blood-brain Barrier Permeability in a Rat Model of Type 2 Diabetes
}

Ju Qiao

Northeastern University College of Science

Christopher M Lawson

Northeastern University College of Science

Kilian FG Rentrup

Northeastern University College of Science

Praveen Kulkarni

Northeastern University College of Science

Craig Ferris ( $\nabla$ c.ferris@neu.edu )

Northeastern University https://orcid.org/0000-0001-9744-5214

\section{Original investigation}

Keywords: quantitative ultrashort time-to-echo, contrast enhanced (QUTE-CE), magnetic resonance imaging, small vessel disease, BBZDR/Wor rat, diabetic encephalopathy, vascular biomarker, ferumoxytol

Posted Date: February 25th, 2020

DOI: https://doi.org/10.21203/rs.2.24439/v1

License: (9) This work is licensed under a Creative Commons Attribution 4.0 International License.

Read Full License

Version of Record: A version of this preprint was published at Journal of Translational Medicine on June 24th, 2020. See the published version at https://doi.org/10.1186/s12967-020-02428-3. 


\section{Abstract}

Background: This is an exploratory study using a novel imaging modality, quantitative ultrashort time-toecho, contrast enhanced (QUTE-CE) magnetic resonance imaging to evaluate the permeability of the blood brain barrier in a rat model of type 2 diabetes with the presumption that small vessel disease is a contributing factor to neuropathology in diabetes.

Methods: The BBZDR/Wor rat, a model of type 2 diabetes, and age-matched controls were studied for changes in blood brain barrier permeability. QUTE-CE, a quantitative vascular biomarker, generated angiographic images with over 500,000 voxels that were registered to a 3D MRI rat brain atlas providing site-specific information on blood-brain barrier permeability in 173 different brain areas.

Results: In this model of diabetes, without the support of insulin treatment, there was global capillary pathology with over $84 \%$ of the brain showing a significant increase in blood brain barrier permeability over wild-type controls. Areas of the cerebellum and midbrain dopaminergic system were not significantly affected.

Conclusion: Small vessel disease as assessed by permeability in the blood brain barrier in type 2 diabetes is pervasive and includes much of the brain. The increase in blood-brain barrier permeability is a likely contributing factor to diabetic encephalopathy and dementia.

\section{Research In Context}

- Angiopathy is an underlying pathology in diabetes affecting numerous organs.

- Increased permeability across brain capillaries is a contributing factor to the pathogenesis of small vessel disease and dementia associated with diabetic encephalopathy.

- Methods for in vivo quantification and localization of changes in blood brain barrier permeability are needed to understand and diagnose the early onset of vascular dementia with type 2 diabetes.

- Tested on a rat model of type 2 diabetes, QUTE-CE provided quantitative measure of blood brain permeability across 173 discrete brain areas.

- The novel imaging protocol, QUTE-CE with ferumoxytol, can be readily used in the clinic to study blood brain permeability and disease progression in diabetic encephalopathy.

\section{Background}

Vascular dementia is a serious consequence of diabetes (1). Prolonged exposure to high blood levels of glucose, typical of type 2 diabetes, affects capillary endothelial structure, function and permeability (2). Failure in the blood brain barrier lies at the foundation of cerebral small vessel disease and contributes to the pathogenesis of diabetic encephalopathy (3). Methods for in vivo quantification and localization of changes in blood brain barrier permeability are needed to understand and diagnose the early onset of vascular dementia with type 2 diabetes. 
Imaging the subtle changes in blood brain permeability is not possible with standard imaging protocols but can be assessed with dynamic contrast enhanced MRI (4). However, dynamic contrast enhanced MRI has several limitations. The concentration versus time curve for gadolinium-based contrast agent is typically $15-30 \%$ inaccurate; therefore, dynamic contrast enhanced (DCE)-MRI has not proven useful clinically (5). It is also difficult to model the effects of contrast agent on both T2* and T1 given the short acquisition time, and strong dependence on microstructural properties such as vessel size, tortuosity and orientation. These and other methodological issues with the use of DCE-MRI for blood brain barrier (BBB) permeability have resulted in significant differences in the reported rates of leakage (5).

To address this issue, a novel imaging modality, quantitative ultrashort time-to-echo, contrast enhanced (QUTE-CE) MRI (6) was used to study changes in blood brain permeability in the BBZDR/Wor rat an inbred rat strain model of type 2 diabetes (7).

\section{Methods}

\section{Animals}

Male Bio-Breeding Zucker diabetic rats (BBZDR/Wor rats) $(n=8)$ and age-matched non-diabetic BBDR littermates $(n=8)$, were obtained from Biomere (Worcester, MA). The obese male BBZDR/Wor rat spontaneously develops type 2 diabetes at approximately 10 weeks of age ( 100\%) when fed standard rat chow. BBZDR/Wor diabetic rat displays all clinical symptoms typically associated with type 2 diabetes including dyslipidemia, hyperglycemia, insulin resistance, and hypertension (7),

Rats were maintained on a $12 \mathrm{~h}: 12 \mathrm{~h}$ light-dark cycle with a light on at 07:00 h, allowed access to food and water ad libitum and were treated with ip injections of saline at indications of weight loss. All animal experiments were conducted in accordance with the Northeastern University Division of Laboratory Animal Medicine and Institutional Animal Care and Use Committee.

(https://academic.oup.com/ilarjournal/article/45/3/292/704910)

Access to rats was dependent upon the breeding schedule and resulting genotypes. This required we run two separate imaging studies, each with four rats from each genotype, separated by six months.

\section{Imaging}

Studies were done on a Bruker Biospec 7.0T/20-cm USR horizontal magnet (Bruker, Billerica, MA, USA)

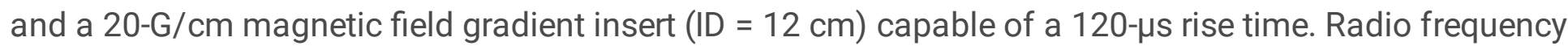
signals were sent and received with a quadrature volume coil built into the rat restrainer (Animal Imaging Research, Holden, Massachusetts). All rats imaged under 1-2\% isoflurane while keeping a respiratory rate of 40-50 breadths/min. At the beginning of each imaging session, a high-resolution anatomical data set was collected using the RARE pulse sequence with following parameters, 35 slice of $0.7 \mathrm{~mm}$ thickness; field of view $3 \mathrm{~cm} ; 256 \times 256$; repetition time [TR] $3900 \mathrm{msec}$; effective echo time [TE] $48 \mathrm{msec}$; number of excitations $3 ; 6$ min $14 \mathrm{sec}$ acquisition time. 
Rats were imaged prior to and following an i.v. bolus of $6 \mathrm{mg} / \mathrm{ml}$ Fe of Ferumoxytol. The injected volume was tailored for each rat (assuming $7 \%$ blood by body weight) to produce a starting blood concentration of $200 \mu \mathrm{g} / \mathrm{ml} \mathrm{Fe}$ ( $2 \times$ the clinical dose approved for use in humans). The QUTE-CE MRI image parameters of $T E=13 \mu \mathrm{s}, T R=4 \mathrm{~ms}$, and flip angle $=20^{\circ}$ utilized a high radio frequency pulse pulse bandwidth of $200 \mathrm{kHz}$. Therefore, the pulse duration was short $(6.4 \mu \mathrm{s})$ compared to the T2 of the approximate ferumoxytol concentration $(4.58 \mathrm{~ms}$ for $3.58 \mathrm{mM}$, i.e. $200 \mu \mathrm{g} / \mathrm{ml}$ to minimize signal blur and reduce the probability for a curved trajectory of the magnetization vector $\mathrm{Mz}$. A $3 \times 3 \times 3 \mathrm{~cm}^{3}$ field-of-view was used with a matrix mesh size of $180 \times 180 \times 180$ to produce $167 \mu \mathrm{m}$ isotropic resolution.

Images were motion-corrected, aligned spatially, and resliced using MATLAB SPM12 toolbox developed at UCL (http://www.fil.ion.ucl.ac.uk/spm/). The pre-contrast UTE images were set as the baseline. For each rat in each imaging session, the voxel wise percentage change of signal intensity for each scan time point (post-con) was calculated as (post-con - baseline)/(blood intensity change) $* 100 \%$ as described in our previous work (10), where blood intensity change is a normalization factor calculated by the post-con blood signal intensity minus baseline blood signal intensity. A 173-region rat brain atlas (Ekam Solutions LLC, Boston, MA, US) was fit to T2-weighted RARE anatomical data set for each rat data set taken at each imaging session, using software developed at Northeastern University Center for Translational Neuroimaging (CTNI), considering the variations in brain size and positions. The fitted atlas was transferred to UTE imaging. Once the images were co-registered to the atlas, custom MATLAB code was used to mask individual brain regions for ferumoxytol measurement.

Mode of percentage change distribution for each of the 173 brain areas for control and BBZDR/Wor rats was statistically compared using a Wilcoxon rank-sum test with the alpha set at 0.05 . Data was analyzed by co-authors Cai and Kulkarni blind to the identity of the groups.

\section{Results}

Shown in Fig 1 is a truncated table from the Rat Brain Atlas of 173 brain areas listing those 26 areas that were not significantly different in blood brain barrier permeability between BBZDR/Wor rats and their littermate controls. The location of these areas can be are visualized in the surrounding $2 \mathrm{D}$ and 3D images generated with the Rat MRI Atlas. All areas in red in the 2D representations show significantly greater blood brain barrier permeability in the BBZDR/Wor rats as compared to controls. Areas in white localized to the prefrontal ctx, midbrain and cerebellum were not significantly different. These areas in white are coalesced into 3D volumes and pictured in the glass brain in yellow.

\section{Discussion}

QUTE-CE MRI, was developed as a quantitative vascular biomarker (6). Ferumoxytol (Feraheme ${ }^{\mathrm{TM}}$ ) MRI with optimized 3D Ultra-Short Time-to-echo (UTE) Pulse Sequences produces angiographic images unparalleled to time-of-flight imaging or gadolinium-based first-pass imaging. The contrast agent is ferumoxytol, an ultra-small superparamagnetic iron oxide nanoparticle with a dextran coating. Since the 
size exceeds the cutoff ( $\sim 6 \mathrm{~nm})$ for glomerular filtration, ferumoxytol is not cleared by the kidney, and instead is an excellent blood pool contrast agent with a long intravascular half-life of $\sim 15 \mathrm{~h} \mathrm{(8)}$. Numerous clinical MRI studies using ferumoxytol have been conducted in children and adults, demonstrating no major adverse effects, thus QUTE-CE can be readily used in the clinic to study BBB permeability (9). We recently published a study mapping the absolute physiological cerebral blood volume (CBV) of the awake rat brain, including measurements of microvasculature density and vascular functional reserve (10). QUTE-CE MRI can be used for identifying hyper- or hypo-vascularization, small vessel density, $\mathrm{BBB}$ permeability and vascular reserve and vascular responsivity to $\mathrm{CO} 2$ challenge at the individual voxel and regional levels using our rat 3D MRI atlas. As demonstrated in this study with the BBZDR/Wor rats, a preclinical model of type 2 diabetes, this imaging technology could be used to diagnose and evaluate blood brain permeability and disease progression in diabetic encephalopathy in the clinic.

\section{Conclusion}

Small vessel disease as assessed by permeability in the blood brain barrier in type 2 diabetes is pervasive and includes much of the brain. The increase in blood-brain barrier permeability is a likely contributing factor to diabetic encephalopathy and dementia.

\section{Abbreviations}

QUTE-CE quantitative ultrashort time-to-echo, contrast enhanced

BBB blood brain barrier

CBV cerebral blood volume

DCE dynamic contrast enhanced

BBBZDR Male Bio-Breeding Zucker diabetic rats

TR repetition time

TE echo time

RARE rapid acquisition with relaxation enhancement

\section{Declarations}

\section{Ethics approval and consent to participate}

not applicable to humans 
All animal experiments were conducted in accordance with the Northeastern University Division of Laboratory Animal Medicine and Institutional Animal Care and Use Committee.

\section{Consent for publication}

not applicable

\section{Availability of data and materials}

All data can be accessed through a link to Mandeley. DOI to follow.

\section{Competing interests}

CFF has a financial interest in Animal Imaging Research, the company that makes the RF electronics and holders for animal imaging.

\section{Funding}

not applicable

\section{Authors' contributions -}

All of the authors have contributed substantially to the manuscript.

Concept, drafting and interpretation - Ferris, Qiao

Execution and analysis - Qiao, Kulkarni, Lawson, Rentrup

\section{Acknowledgements}

We thank Biomere (Worcester, Massachusetts, USA) for providing the BBZDR/Wor rats and their agematched non-diabetic BBDR littermates for these studies.

\section{References}

1. Pasquier, F., Boulogne, A., Leys, D., and Fontaine, P. (2006) Diabetes mellitus and dementia. Diabetes Metab 32, 403-414

2. Allen, C. L., and Bayraktutan, U. (2009) Antioxidants attenuate hyperglycaemia-mediated brain endothelial cell dysfunction and blood-brain barrier hyperpermeability. Diabetes, obesity \& metabolism 11, 480-490

3. Bogush, M., Heldt, N. A., and Persidsky, Y. (2017) Blood Brain Barrier Injury in Diabetes: Unrecognized Effects on Brain and Cognition. Journal of neuroimmune pharmacology : the official journal of the Society on Neurolmmune Pharmacology 12, 593-601 PMC5693692 
4. Starr, J. M., Wardlaw, J., Ferguson, K., MacLullich, A., Deary, I. J., and Marshall, I. (2003) Increased blood-brain barrier permeability in type II diabetes demonstrated by gadolinium magnetic resonance imaging. Journal of neurology, neurosurgery, and psychiatry 74, 70-76

5. Raja, R., Rosenberg, G. A., and Caprihan, A. (2018) MRI measurements of Blood-Brain Barrier function in dementia: A review of recent studies. Neuropharmacology 134, 259-271

6. Gharagouzloo, C. A., McMahon, P. N., and Sridhar, S. (2015) Quantitative contrast-enhanced MRI with superparamagnetic nanoparticles using ultrashort time-to-echo pulse sequences. Magn Reson Med 74, 431-441

7. Tirabassi, R. S., Flanagan, J. F., Wu, T., Kislauskis, E. H., Birckbichler, P. J., and Guberski, D. L. (2004) The BBZDR/Wor rat model for investigating the complications of type 2 diabetes mellitus. ILAR J 45, 292-302

8. Bremerich, J., Bilecen, D., and Reimer, P. (2007) MR angiography with blood pool contrast agents. European radiology 17, 3017-3024

9. Muehe, A. M., Feng, D., von Eyben, R., Luna-Fineman, S., Link, M. P., Muthig, T., Huddleston, A. E., Neuwelt, E. A., and Daldrup-Link, H. E. (2016) Safety Report of Ferumoxytol for Magnetic Resonance Imaging in Children and Young Adults. Investigative radiology 51, 221-227

10. Gharagouzloo, C. A., Timms, L., Qiao, J., Fang, Z., Nneji, J., Pandya, A., Kulkarni, P., van de Ven, A. L., Ferris, C., and Sridhar, S. (2017) Quantitative vascular neuroimaging of the rat brain using superparamagnetic nanoparticles: New insights on vascular organization and brain function. Neurolmage 163, 24-33

\section{Figures}




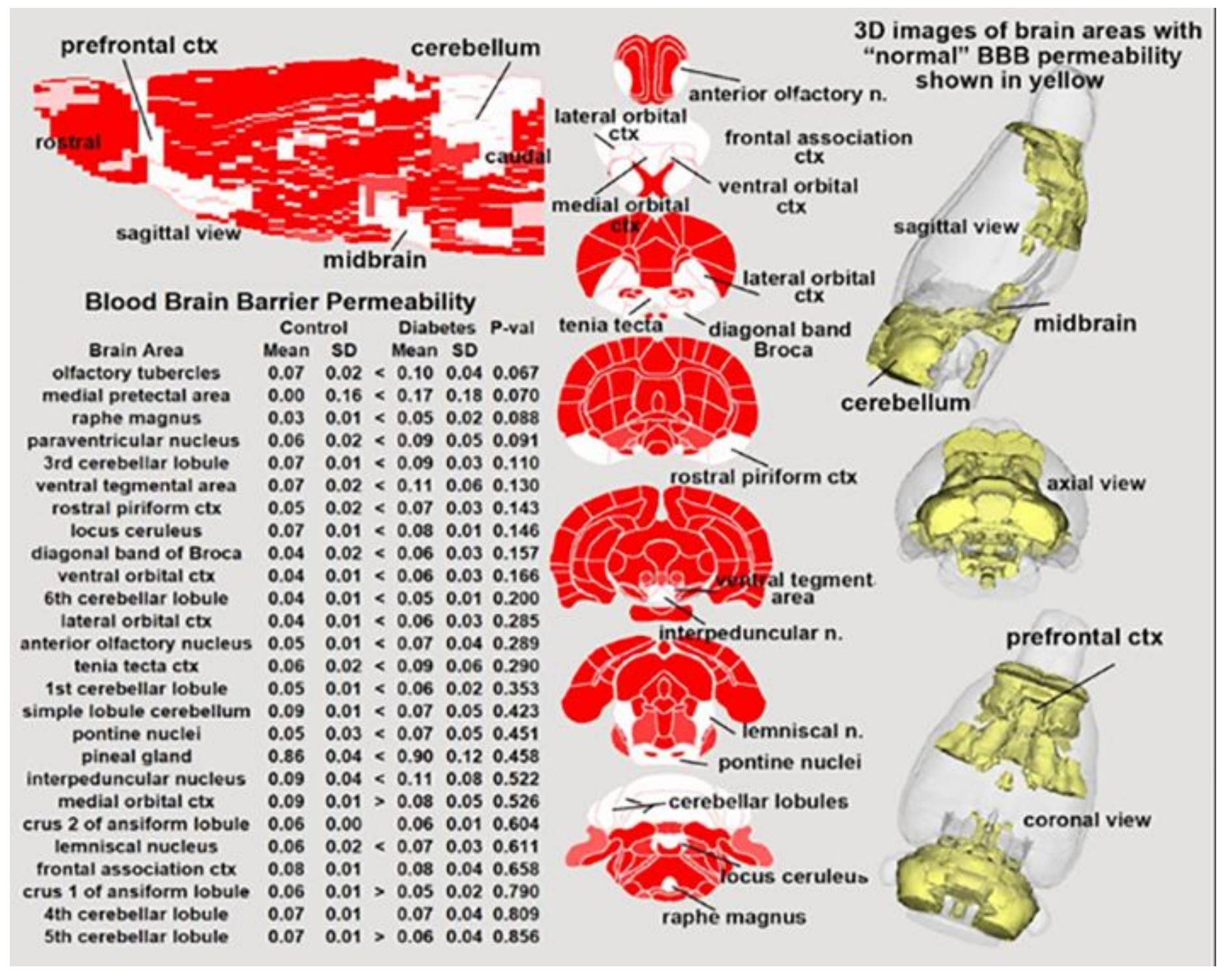

Figure 1

Imaging Blood Brain Barrier Permeability. Quantitative Ultra-Short Time-to-Echo, Contrast Enhanced imaging of blood brain barrier permeability comparing BBZDR/Wor rats and their littermate controls. All areas in red show significantly greater permeability in type 2 diabetic as compared to controls. 